\title{
XVI.
}

Aus der psychiatrischen Klinik der Universität Würzburg.

\section{Ein Beitrag zur Kenntniss der Brückengeschwïlste.}

\author{
Von \\ Dr. Theodor Zahn, \\ Assistent der Klinik.
}

Die folgenden Ansführungen dürften ausser dem anatomischen Interesse, das die ausgedehnte im Rückenmark und Hirnstamm gefundene syphilitische Erkrankung bietet, besonders deswegen Beachtung verdienen, weil die dabei in der Brücke aufgetretene, nebenbei einzige, Gummigeschwulst ohne jede Raumverdrängung die schwersten Hirndrucksymptome verursachte. Sodann giebt dieses Beispiel Anlass zu einer Erörterung des wirklichen Entstehungsortes der cerebellaren Ataxie bei Brückengeschwülsten.

Krankengeschichte.

Die 28jährige B. S., eine unter polizeilicher Aufsicht stehende Puella publica, wurde am 28. März 1900 in die psychiatrische Klinik gebracht mit der bezirksärztlichen Notiz: „Leidet an Verwirrtheit, fällt öfters zu Boden." Sie blieb nun 8 Tage in der Klinik. Hier wurde nur bemerkt, dass sie beim Gehen häufig etwas hin- und herschwankte. Sonst zeigten sich keine auffälligen psychischen oder körperlichen Störungen. Nur schien die Eingelieferte sehr dumm, halb idiotisch zu sein. Das. war sie jedoch offenbar von Hanse aus, wie unter Anderem ihre früheren Schulzeugnisse bewiesen. Zeichen von Lues waren keine zu entdecken. Der Urin war frei von Zucker, enthielt etwas Eiweiss und zeigte das Vorhandensein eines Blasenkatarrhs an. Das Eiweiss verschwand bald wieder, wie gleich bemerkt sei; der Blasenkatarrh blieb dagegen auch in der Folgezeit bestehen:

Die Kranke äusserte selbst über ihre letzte Vergangenheit: „Ieh bin immer so dumm und schwindelig geworden; ich seh' Alles drei- und viermal und lasse Alles fallen. Ich bin auch ein paar Male zu Haus hingefallen."

Eine Diagnose war während dieses ersten Aufenthalts nicht zu stellen. Für Epilepsie lagen keine genügenden Anhaltspunkte vor, ebensowenig für eine Nierenerkrankung. Nirgends waren bestimmte Innervationsstörungen nachweisbar. Zwar schien eine Verstärkung der beiderseitigen Patellarreflexe zu bestehen, konnte jedoch nicht sicher behauptet werden. Zuweilen liess sich auch Fussclonus rechts und links hervorrufen, zu anderen Zeiten aber wieder nicht. Diese zunächst noch undeutlichen Punkte erhielten 
später durch die Degenerationen der Pyramidenseitenstränge im Rückenmarksbefund eine hellere Beleuchtung.

Weil sich in der Klinik nichts Abnormes von grösserer Bedeutung. herausgestellt hatte, wurde die S. nach Hause zurückgegeben, am 3. April. Doch schon nach 2 Tagen warde sie wegen schlechter Aufführung wieder eingeliefert. Anch während des zweiten Anfenthalts vom 5. April bis 4. Mai war ihr psychisches Verhalten, von dem Schwachsinn abgesehen, nicht ungewöhnlich. Der Gang war wie das letzte Mal oft schwankend. Dagegen wurde eine Störung der Facialisinnervation neu entdeckt. Die rechte Gesichtsseite war besser innervirt. Diese Unregelmässigkeit war jedoch so unbedentend, dass man glaubte, an die Möglichkeit einer angeborenen Asymmetrie denken zu müssen, wie sie häufig beobachtet wird. Dieselbe wäre das erste Mal vielleicht nicht genügend beachtet worden. Doch lehrte im weiteren Krankheitsverlaufe das Zunehmen dieser Erscheinung, dass es sich um eine beginnende Lähmung hier handelte.

Am 4. Mai entlassen, wurde die Person aus dem gleichen Grunde wie das vorige Mal am 11. Mai wieder eingebracht. Nunmehr war doch eine beginnende Hirnkrankheit nicht mehr zu verkennen. Die Patientin litt vor Allem viel an Schlafsucht. Dazwischen hinein war sie wieder munter und lebhaft und schwatzte sehr viel, übrigens ohne eine Spur von Sprachstörung bezïglich des Inhalts und der Articulation.

Wegen Verdachts auf eine Hirngeschwulst wurde eine genaue ophthalmoskopische Untersuchung vorgenommen (von Herrn Prof. Dr. Bach). Dabei fand sich in keinem Auge eine ausgebildete Staungspapille, nur venöse Hyperämie im Augenhintergrunde; rechts mehr als links. Die Papillengrenzen waren unverwischt. Wegen vorhandenen Schwindels und Gefühls von Trunkenheit wurde auch das Hörorgan näher untersucht; es war functionell and anatomisch keine Störung auffindbar. Anch war wie das Sprechdas Schreibvermögen gut erhalten.

Eine bestimmte Localdiagnose war demnach noch nicht möglich. $\mathrm{Zn}$. eventueller antiluetischer Behandlung wurde die Kranke am 28. V. in die Syphilisklinik übergeführt. An diesem Tage war sie bei vollem Bewnsstsein so heftig hingefallen, dass sie sich eine starke Beule am Hinterkopf zugezogen hatte.

In ihrer nenen Umgebung führte sich die Patientin aber so abscheulich auf, dass sie schon am 30. V. der psychiatrischen Klinik zurückgegeben wurde. Dem Versuche, sie einer Schmierkur zu unterziehen, hatte sie wilden Widerstand entgegengesetzt. Von jetzt an blieb ihr Zustand bis zum 8. VII. $1900 \mathrm{im}$ Wesentlichen der gleiche. Sie schwankte stark beim Gehen, stürzte oft zu Boden, ohne dass man hierbei eine regelmässige Bewegungsrichtung hätte wahrnelmen können. Auch war sie oft schlafsüchtig und abwechselnd wieder zum Schimpfen und Schreien aufgelegt. Zuweilen ging sie auch wieder umher, wobei ihr Gang nichts Auffälliges zeigte, also frei von Bewegungsataxie war. Noch immer fehlten scharf ausgeprägte Innervationsstörungen. Im Besonderen waren die Facialislähmung sowie der Befund im Augenhintergrunde noch so geringfügig wie früher. Der letztere änderte sich auch bis zum Tode nicht. Es sei hier beigefügt, dass auch nur einigermassen verwerthbare Untersuchungen des sensiblen und elektrischen Verhaltens der Nervengebiete bei der ungeberdigen Patientin ausgeschlossen waren. 
Am Morgen des 8. Juli brach sie völlig zusammen und war fortan gar nicht mehr im Stande zu gehen oder zu stehen. Sie lag andauernd in comatösem Zustande da, ohne ein Wort zu reden. Die linke Gesichtshälfte war jetzt zweifellos von einer Lähmung befallen, woran auch das linke Ange durch vermindertes Schliessungsvermögen Theil nahm. Ferner war der linke Arm deutlich gelähmt; er war ganz schlaff und leblos. Mit dem rechten dagegen machte die Patientin noch Bewegungen. Die Beine waren beide in gleichem Maasse schlaff und reglos; es konnte hier nichts Genaueres mehr festgestellt werden. In den letzten Lebenstagen trat noch einige Male Erbrechen auf. Auch stieg in den beiden letzten Tagen vor dem Tode die früher normale Körpertemperatur auf $40^{\circ}$ und blieb daranf. Nach zehntägigem tiefem Coma starb die Patientin am 17. Juli 1900.

\section{Anatomische Untersuchung.}

Bei der Hirnsection wurde anfgezeichnet:

Pia normal, etwas schwer abzuziehen.

Windungen etwas abgeplattet, die Furchen flach.

Gewicht des Gehirns mit Häuten $1212 \mathrm{~g}$. Herd im Pons links, überschreitet nicht die Mittellinie. An den Blutgefässen nichts Besonderes. Von einem auffälligen Befund am übrigen Körper ist nichts vermerkt.

Hirnstamm und Rückenmark kamen nun in Formol, später in Müller'sehe Flüssigkeit. Dieser entnommen weist das Rückenmark von oben bis in den Sacralabschnitt helle Stellen im Gebiete der PyS und KIS auf, rechts viel auffälliger, als links. Der Herd in der Brücke sitzt ventral in deren hinteren Hälfte, an die Medianlinie stossend. Er ist von grauer Farbe. Seine Oberfläche ist in der Mitte leicht eingesunken. Sie bildet ein Quadrat von $12 \mathrm{~mm}$ Seitenlänge. Die rechte Brückenhälfte scheint ganz frei zu sein.

Auf einem frontalen Schnitt durch den Brückenabsehnitt (sammt Haubenfeld), etwa in der Mitte des Herdes, ist dieser $4 \mathrm{~mm}$ tief. Die Brückenoberfläch $\theta$ ist durch ihn nicht vergrössert; die neue Masse hat sich augenscheinlich auf Kosten der links grösstentheils untergegangenen Pyramidenbahnen entwickelt. Es sind nicht einmal die queren annähernd geradlinigen Brückenfasern durch die Neubildung dorsalwärts ausgebogen worden. Die Höhe des Brïckenabschnitts an der dicksten Stelle des Herdes beträgt $19,5 \mathrm{~mm}$, genau wie an der entsprechenden Stelle rechts. Um die Neubildung herum ist das Gewebe erweicht und lückenhaltig.

Von der Brücke und dem oberen Theile des verlängerten Marks wurden Schnitte in fortlanfender Serie angefertigt, die nur durch die Wegnahme eines kleinen Stückes zur M ar chi-Färbung unterbrochen wurde. Vom Rückenmark wurde aus allen Höhen eine grössere Anzahl von Präparaten gemacht. Färbung: zumeist Weigert'sche Markscheidenfärbung, ferner nach Marchi, mit Carmin, mit Hämatoxylin-Eosin.

Beschreibung des Herdes. In den Weigert-Präparaten bleibt von der linken Hälfte des Brückenabschnitts das ventralste Viertel ganz ungefärbt, ferner ein schmales Stïck vom Rande in grösserer Ausdehnung. Der Herd stösst dorsalwärts an eine schmale quere Lücke mit zerfetzter Wandung. In der $\mathbf{r} \theta \operatorname{chten}$ Brückenhälfte ist ein kleiner medial und ventral liegender Theil der Pyramidenbahnen degenerirt, ausserdem ein schmales Stück des ganzen ventralen Randes. In den degenerirten Stellen fehlen 
die Yarkfasern völlig, in den anstossenden sind sie theilweise schwach gefärbt. Man findet hier auch mit Hülfe der Marchi-Färbung frisch degenerirte Marksubstanz. Die Degeneration der Pyramidenbahnen lässt sich cerebralwärts bis in den obersten Theil der Brïcke, spinalwärts ununterbrochen durch das verlängerte Mark und die Pyramidenkreuzung in die entsprechenden Pyramidenseitenstränge rechts und links verfolgen, und zwar bis in den Sacralabschnitt. Die Degeneration wird unterhalb der Brücke bald leichter und drückt sici durch geringere Färbung als in den normalen Partien aus.

In dem Degenerationsherde der Brücke sind zwischen die wohl erhaltenen Gliazellen in die Grundsubstanz ausserordentlich zahlreiche stark gefärbte Rundzellen eingelagert, theils mehr diffus, theils dicht zusammengedrängt (bei Alaunhämatoxylin-Eosinfärbung). Dazwischen trifft man auch grössere ovale oder spindelige Zellen, wie Fibroblasten aussehend. Das infiltrirte Gewebe geht rechts allmählich in das nicht infiltrirte über, links bilden streckenweise die oben erwähnten Höhlen die Begrenzung. Die Gefässe sind zum Theil erweitert und enthalten viele Rundzellen in ihrer Adventitia.

Es handelt sich also um ein flächenhaft ausgebreitetes Granulationsgewebe mit erheblicher Verdickung in der linken Brückenhälfte und Erweichung in der Umgebung dieser Verdickung. Die Leukocyteninfiltration reicht in abgeschwächtem Grade bis ins oberste Brückenende. In den Hirnschenkelfiussen liegen nur noch um die Gefässe, allerdings theilweise beträchtliche Rundzellengruppen. Spinalwärts trifft man im proximalen Ende der Medulla oblongata links noch mässige diffuse Rundzellenansammlungen vornehmlich im degenerirten Theile der Pyramidenbahnen, rechts nur noch in der Umgebung einzelner Gefässe. Im unteren Abschnitte des verlängerten Marks sind auch links nur noch vereinzelte Infiltrationen an kleinen Gefässen $\mathrm{zu}$ sehen.

Was den mehr dorsal gelegenen Theil des Brückenquerschnitts anbelangt, so ist in dessen mittlerer Höhe nichts Besonderes zu verzeichnen. Unter der Ventrikeloberflache dagegen sind die Gefässe vielfach erheblich erweitert, ihre Wandung zuweilen infiltrirt and verdickt. Sie liegen in ausgeweiteten Gefässscheiden. Um die Gefässe sind hier und dort Rundzellen angehäuft. Es sind sowohl kleine Arterien als Venen betheiligt. Die Leukocyteninfiltration betrifft vorwiegend die Adventitia, weniger die Media und gar nicht die Intima.

Die Pia ist an der Brücke nicht mit erhalten. Am verlängerten Mark dagegen ist ein Stückchen haften geblieben und mitgeschnitten worden. Es ist erheblich verdickt und infiltrirt, enthält infiltrirte Gefässe.

Von den Gehirnnerven der Brücke und des verlängerten Marks ist zu sagen:

Die Faserbündel und Kerne des linken Hypoglossus, Vagus, Glossopharyngeus sammt dessen spinaler Wurzel sind gut ansgebildet und deutlich zu sehen.

Ebenso sind vom Acusticus der dreieckige, grosszellige und accessorische Kern, sowie die spinale, mediale und laterale Wurzel und die Striae medullares wohl entwickelt

Vom l. Facialis sind der Kern und der Kernschenkel nicht auffindbar, dagegen sind das quere Zwischenstück und der ventral und lateral ver- 
laufende Austrittsschenkel in Gestalt eines kräftigen Bündels bis in die Nähe des Herdes zu verfolgen. Hier wird das Bündel lichter und enthält Spuren frischer Degeneration.

Den Abducens zu erkennen, gelang nicht. Höchst wahrscheinlich lag er zwischen der oberen Reihe von Weigert-Präparaten und den MarchiSchnitten, welche Unterbrechung beim Mikrotomiren nicht ohne Verlust abging. Seinem Verlaufe nach musste er durch den Herd ziehen.

Der Trigeminus ist mit seiner spinalen und cerebralen Wurzel, mit seinem sensiblen und dem grosszelligen motorischen Kern kräitig entwickelt.

Auch im Uebrigen sind an der grauen Substanz und den Markfasern der Brücke und der Med. obl. rechts und links keine Veränderungen nachzuweisen.

Im Besonderen ist zu bemerken, dass die Corpora restiformia beiderseits kräftig entwickelt sind. Zu ihnen ziehen in der obersten Höhe der Schleifenkreuzung die ànsseren Bogenfasern aus der Gegend der KIS in starken Zügen hin, während die Pyramiden gelichtet sind. In der Höhe des 1. Cervicalnerven ist aber auch das Feld der KlS nur schwach gefärbt, allerdings weniger schwach als die PyS.

Rückenmark. In den Seitensträngen trifft man von oḅen bis ins Sacralmark rechts wie links Degenerationen. Dieselben sind rechts mittleren Grades, unten schwerer als oben. Links sind sie durchwegs leichter. Im mittleren Halsmark z. B. sind sie hier nur eben noch erkennbar. Doch werden sie auch hier nach unten zu stärker. Die Degenerationen nehmen auf dem Querschnitte das ganze Gebiet ein, das hinter einer durch den Centralkanal gelegt gedachten Querlinie liegt, also auffallender Weise auch die KlS. Und zwar verhält es sich so über den ganzen Verlauf der KlS nach unten hin. Die seitlichen Grenzschichten bleiben rechts und links frei. Ebenso die Vorderstränge. Nach den Marchi-Präparaten zu schliessen, sind die Degenerationen nicht frisch.

Die Hinterstränge sind durchgehends tief dunkel gefärbt; wie auch die hinteren Wurzeln beim Austritt.

Die grane Substanz erscheint mit ihren Zellen nnd Fasern überall normal. Speciell auch die Clarke'schen Sänlen.

Die Neuroglia ist in den Degenerationsfeldern compensatorisch vermehrt. Die Rindenschicht ist nicht verbreitert.

Die beiden Blätter der Pia sind über das ganze Rüekenmark hin verdickt, theils infiltrirt, theils fibrös. Diese Veränderung ist weder auf dem einzelnen Querschnitte noch anf den verschiedenen Höhen gleichmässig ausgeprägt. An einzelnen Stellen ziehen Gruppen von Rundzellen in die Spalten des Rückenmarks von der Oberfläche her hinein.

Ein auffallendes Verbalten zeigen die Gefässe der Pia und der Rückenmarkssubstanz. Hauptsächlich im Brustabschnitt, in geringerem Maasse weiter oben und noch weniger weiter unten. Viele Gefässe sind stark erweitert; einige in der weissen und grauen Substanz sind für das blosse Auge leicht sichtbar geworden. Die Wand mancher Pialvenen ist erheblich verdickt und reich mit rundlichen und spindeligen Zellen durchsetzt. Rundzelleneinlagerungen sind auch in anderen Gefässen, kleinen Arterien und Venen, vielfach zu sehen. Im Wesentlichen ist die Adventitia davon betroffen. Die Wand sieht auch häufig etwas verdünnt ans. Obliterationen 
fehlen. Die Erweiterungen finden sich in allen Theilen des Querschnitts, in der weissen wie in der granen Substanz, am wenigsten in den Hintersträngen.

Man erhält nicht den Eindruck einer ursächlichen Beziehung zwischen den Gefässstörungen und den Degenerationen. Zwar sind in deren Gebieten besonders viele weite Gefässe, allein es hat mehr den Anschein, als habe das gelockerte Gefüge der Marksubstanz die Erweiterungen hier begünstigt. Die Degenerationsgebiete sind sogar stellenweise geradezu netzartig gelockert. Ferner sind in den Halspartien in den stark degenerirten Theilen nur ganz wenig Erweiterungen und andererseits weiter unten auch in den normalen Theilen viele Erweiterungen. Anch in hinteren ausgetretenen Wurzelbündeln mit wohl erhaltenen Markfasern liegen hier und dort erweiterte Gefässse.

Beurtheilung der anatomischen und klinischen Beobachtungen.

Es ergiebt sich aus dem Vorstehenden eine vom Sacralmark bis zum Hirnstamm verbreitete Entzündung der Pia mit Verdickung und Rundzelleneinlagerung. Ferner an der ventralen Brückenoberfläche ein Granulationsherd, der an seiner dicksten Stelle in der linken Brückenhälfte zu Erweichung seiner Umgebung geführt hat. Im Bereiche dieser Neubildung sind die Pyramidenbahnen zu Grunde gegangen. Diese Degeneration ist ununterbrochen in die Pyramidenbahnen des Rückenmarks bis an deren Endigung herabgestiegen. Weiterhin sind die Gefässe des Marks und des Brückenabschnitts, sowohl in der Pia als in der Nervensubstanz selbst, in erheblicher Zahl erweitert und besitzen verdickte oder verdünnte Wandungen mit Rundzelleninfiltrationen. Auch liegen um einzelne Gefässe Gruppen von Rundzellen. Die grösseren Gefässe sind anscheinend unbetheiligt.

Die Natur dieser Gefäss- und Pia-Veränderungen muss als s y philitisch, der Granulationsherd als Gumma angesprochen werden. Dafür geben das Vorleben der Verstorbenen und das gleichzeitige diffuse Befallensein der Pia und der Gefässe den Beleg. Es darf wohl angenommen werden, dass ähnlich wie im Rückenmark von der Pia aus die Rundzellengruppen ins Innere eindrangen, so an der Brücke eine massenhaftere Wucherung von solchen zunächst an der Oberfläche erfolgte.

Um übrigens hier einen Tuberkel sicher ausschliessen zu können, wurde der Granulationsherd auch auf Tb-Bacillen untersucht; wie von vorneherein zu erwarten stand, mit verneinendem Ergebniss.

Bei der syphilitischen Gefässveränderung ist beachtenswerth, dass von den Verdickungen und Leukocytenanhäufungen nur die Media, Adventitia und die Ungebung der Gefässe betroffen sind. Die 
Intima ist nirgends verdickt, das Lumen ist nirgends obliterirt. Dieser Befund steht der Heubner'schen Anschaung ron der Intima als Ausgangspunkt des syphilitischen Gefässprocesses entgegen. Er bestätigt vielmehr die neuere für einen Theil der Fälle gültige Ansicht, die von Baumgarten, Obermeyer u. A. vertreten wird und auch von Weygandt ${ }^{1}$ ) durch ein Beispiel illustrirt wurde, wonach in einer Mesound Perivasculitis der Anfang der Erkrankung gefunden wird.

Das Alter der syphilitischen Affection des Centralnervensystems lässt sich nicht genau bestimmen. Klinische Erscheinungen hatte die nicht eben hochgradige Rückenmarkssyphilis nicht gemacht. Die ersten Symptome seitens der Gummigeschwulst im Pons, die cerebellare Ataxie, wurden etwa 4 Monate vor dem Tode bemerkbar. Sicherlich war damals das Gumma bei Weitem nicht so gross and dicht wie später. Der Facialis, dessen Lauf beim Verlassen der Brücke durch. den Herd ging; war anfangs nur ganz leicht beschädigt. Die grössere Ausdehnung des Granulationsherdes und die Erweichung seiner Umgebung scheinen ziemlich frisch zu sein. Das Vorherrschen der massenhaften Rundzellen, die mässige Schwere der offenbar älteren Degenerationen in den Pyramidenseitensträngen, endlich die lang erhaltene Bewegungsfähigkeit auch der rechten Extremitäten lassen das vermuthen.

Die periphere Facialislähmung der linken Gesichtshälfte, die in der Zeit vor dem Tode deutlicher als früher geworden war, erhält ibren anatomischen Ausdruck in dem Faserschwund und in den Markschollen an der Austrittsstelle.

Die Extremitäten waren vor Eintritt des Comas nicht gelähmt. Der Gang der Patientin war, von der Gleichgewichtsstörung abgesehen, unbehindert. Dies widerspricht nicht dem Degenerationsbefund in den Pyramidenbahnen des Rückenmarks. Noch stärkere Degenerationen in denselben gehen bekanntlich nicht immer mit sichtbaren Bewegungsstörungen einher. Die gleichen Degenerationen geben dagegen für die gesteigerten Sehnenreflexe eine Bestätigung und $\mathrm{Er}$ klärung. Diesem Symptom auf der gelähmten oder auf beiden Seiten begegnet man in Berichten über Ponstumoren nicht gerade häufig. Zudem ist fast nie ein Rückenmarksbefund erhoben. Unser Beispiel weist aufs Neue auf die Nothwendigkeit dieser Untersuchung hin.

In der linken Brückenbälfte waren die Pyramidenbahnen grösstentheils zu Grunde gegangen. Nur ein dorsal gelegener, die ganze Quere einnehmender Rest war noch übrig geblieben. Es muss darum auffallen, dass der rechte Arm in der letzten Zeit besser bewegt werden konnte, als der völlig gelähmte linke. In der rechten Brückenhälfte hatten die Pyramidenbahnen nur wenig an Zahl eingebüsst. 
Allerdings stimmt die Degeneration des medialen Theils hier mit der Annahme überein, dass die Pyramidenbahnen für die Arme medial verlaufen. Bei der linken Brückenhälfte muss man sich mit der Thatsache zu begnügen suchen, dass eben nicht alle ihre Fasern fehlen und dass erfahrungsgemäss die klinischen und anatomischen Störungen bei solchen Degenerationen einander oft nicht genau zu entsprechen scheinen.

Wie der Verlauf der Erkrankung lehrt, starb die Patientin unter den Zeichen schweren Hirndrucks nach zehntägigem Coma. Vorher hatten schon die grosse Schlafsucht, die Verwirrtheit und der Schwindel darauf hingewiesen. Auch war im Augenhintergrund die venöse Hyperämie gefunden worden; doch darf auf diese kein besonderes Gewicht gelegt werden.

Die zweifellosen Hirndrucksymptome stehen scheinbar in schroffem Widerspruch zu der anderen Thatsache:

Das Gumma konnte vermöge seines Umfanges nicht die geringste Raumverdrängung im Gehirn verursachen, da es sich auf Kosten der Hirnsubstanz entwickelt hatte.

Ausser dem Gumma war jedoch kein anderer Grund für die Hirndruckerscheinungen zu entdecken.

Da also das Gumma augenscheinlich doch diese Erscheinungen veranlasst hatte, nur nicht unmittelbar durch Raumverdrängung, so muss zwischen Gumma und Hirndruck eine Vermittelung stattgefunden haben.

Eine solche kann am besten der Medulla oblongata zugeschrieben werden.

Diese lag erstens im Einflussbereich des nahen Gummas. Zweitens lässt sie leicht die Annahme der Erzeugung von Circulationsstörungen im Gehirn zu, wodurch Drucksymptome entstehen konnten. In der Medulla oblongata sitzt ja das Centrum für die Vasomotoren (beim Kaninchen vom oberen Theile der Rautengrube bis $3-4 \mathrm{~mm}$ oberhalb des Calamus scriptorius), zugleich auch des Gehirns. Bei Lähmung dieses Centrums muss es zu Hyperämie und. Schwellung des Gehirns und folglich zu Druckerscheinungen kommen. Zum Beleg hiefür lassen sich die ganz in dieser Richtung gehenden Versuche Spina's ${ }^{2}$ ) anführen. Darnach erhöht die Durchschneidung des verlängerten Marks bei Hunden den Bluitdruck im Gehirn durch Lähmung der Vasoconstrictoren der cerebralen Gefässe. So wird das Gehirn mit Blut überfüllt. Bei Anlegung einer künstlichen Schädelöffnung kann man an den vorliegenden Hirntheilen Röthung; erhebliche Quellung, sogar Prolaps sehen.

Diese Ueberlegungen erhalten nun in unserem Falle eine sinn- 
fällige und kräftige Stütze in Folgendem. An dem Gehirn waren laut Sectionsprotokoll die Windungen abgeplattet, die Furchen flach. Für die hierin ansgesprochene Hirnvergrösserung ist ein weiterer Beweis die abnorm geringe Differenz zwischen der Inhaltszahl des macerirten Schädels und der Gewichtszahl des Gehirns (1280 ccm und $1212 \mathrm{~g})=68$, in Procenten 6. An der Hand zahlreicher sorgfältiger Hirnwägungen und Inhaltsbestimmungen macerirter Schädel nach einer hier nicht weiter auszuführenden Methode fand Rieger eine durchschnittliche procentuale Differenz zwischen jenen beiden Zahlen von 10. Dass hierbei das specifische Gewicht des Gehirns, in unserem Falle 1037, vernachlässigt und die Gewichtszahl mit der Inhaltszahl verglichen werden dürfen, hat Zanke ${ }^{3}$ ) schon dargelegt.

Das Gehirn füllte also diesmal den Schädelraum mehr als gewöhnlich aus. Es war statt um 10 Proc. nur um 6 Proc. kleiner als dieser. Hydrocephalus war nicht vorhanden. Die Vergrösserung des Gehirns kann hier keinen anderen Grund als in einer Schwellung, Hypertrophie, haben.

Eine ähnliche Hirnschwellung bei Brückengescbwülsten sehe ich nirgends erwähnt. Ich halte es aber doch für möglich, dass eine solche in manchen Fällen die vorhandenen. Druckerscheinungen zum grossen Theil verschuldet habe. Bei solchen Ponstumoren nämlich deren Grösse an sich nicht zur Herstellung eines erheblichen Druckes auszureichen erscheint. Findet man, dass eine Brückengeschwulst schon bei geringerer Grösse zu Hirndruck führen kann als eine Geschwulst in anderen Hirntheilen, und dass sie so klein sein kann, dass ihre Masse als solche den Druck nicht wohl allein zu erzeugen vermag, so muss der Gedanke an eine Hirnschwellung, veranlasst von der benachbarten Medulla oblongata, auftanchen. In der That stösst man bei Durchmusterung vieler Fälle von Hirngeschwülsten verschiedene Male auf die Angabe, dass ein Tumor im Pons von nicht mehr als Erbsengrösse zu Hirndrucksymptomen geführt habe (Delbanco's ${ }^{4}$ ) Fälle 30 u. 31; Wiersma, Neurol. Centralblatt 1900 S. 728). Es wäre schwer sich vorzustellen, dass eine so kleine Neubildung an sich, an einem schwereren Hirndrucke schuld gewesen wäre. Und in anderen Hirngegenden ist dies auch wirklich nicht der Fall. Hier sind die Geschwülste, die merkliche Erscheinungen im Leben gemacht haben, alle beträchtlich grösser, wie aus verschiedenen Zusammenstellungen zu entnehmen ist. Sicherlich bleiben viele Hirngeschwülste unbeachtet, weil sie die von ihrer Stelle aus wirksame Grösse nicht erreichen.

Ueberaus heftig war bei der Kranken das Symptom des Schwankens und Taumelns ausgebildet, wodurch sie häufig schlimm zu 
Falle kam. Der gleichzeitige Schwindel sowie das Gefühl des Berauschtseins waren nicht Ursache, sondern Begleiterscheinung des Taumelns und dürften den nämlichen Entstehungsgrund haben. Von der cerebellaren Ataxie bei Ponstumoren sagt Oppenheim ${ }^{6}$ ) (Die Geschwülste des Gehirns. S. 156), dass sie öfter beobachtet werde als die Bewegungsataxie. "Beachtenswerther Weise handelt es sich in fast allen diesen Fällen um Geschwülste, die sich nicht scharf auf das Terrain der Brücke beschränken, sondern auf die Kleinhirnstiele oder das Kleinhirn übergreifen, beziehungsweise diese Theile durch Druck in Mitleidenschaft ziehen."

Unter dem Eindruck, den ich durch die Kenntnissnahme einer grösseren Anzahl von Ponstumoren erhielt, muss ich ebenfalls bei ihnen der Ponsläsion selbst die Schuld an der cerebellaren Ataxie absprechen, und zwar nicht in den meisten, sondern in allen Fällen. Ausserdem glaube ich eine Mitbetheiligung nicht nur des Kleinhirns und seiner Stiele, sondern auch der benachbarten Medulla oblongata ins Auge fassen zu müssen, deren Läsion, wie sich 0 ppenheim ausdrückt (1. c. S. 163), sehr wahrscheinlich unmittelbar cerebellare Ataxie veranlassen kann.

Unter den 30 von Bernhardt ${ }^{7}$ gesammelten Fällen von Ponstumoren führten $5 \mathrm{zu}$ unsicherem und schwankendem Gang $(9,15$, $25,29,30)$. In allen diesen zeigt das klinische oder anatomische Bild, dass ein starker Druck von der Neubildnng ausgeübt wurde; zweimal wird bei der Section die Compression, beziehungsweise Mitbetheiligung des Kleinhirns direct gesehen. In einem 6. Falle (18) mit Unvermögen zu gehen erstreckte sich die Geschwulst gleichfalls auf das Kleinhirn.

Von 6 weiteren bei Nothnage ${ }^{8}$ ) angeführten Fällen von Ponstumoren gehört der von Ladame hierher (S. 123), bei dem wieder das Cerebellum gedräckt zu sein scheint. Dagegen handelt es sich in dem Falle Penzoldt's (S.125) mit Schwanken und Neigung zum Rück. wärtsgehen um einen etwas mehr als erbsengrossen Tuberkel im hinteren Ponsdrittel, auf welchen Penzoldt diese Symptome bezieht. Hier ist offenbar eine Wirkung auf die Medulla oblongata ungezwungen denkbar.

Delbanco hat 46 Beschreibungen von Brückengeschwülsten vereinigt. Davon hatten 6 cerebellare Ataxie zur Folge $(1,23,25,30$, 32, 39). Ueberall hatten hier erhebliche Druckerscheinungen im Leben bestanden. Bei 3 fanden sich zudem die Crura cerebelli mitergriffen. Der Verfasser selbst weist auf den Schwindel als Ursache in allen diesen Fällen hin.

Sonst fand ich noch 19 Beschreibungen von Brückengeschwülsten 
in der Literatur zerstreut, meist im Referat mir zugänglich. Unter diesen sind wieder 6 mit cerebellarer Ataxie verbunden (Kolisch, Diller, Handford, Hofmann, Borowikow, Strözewski). Dieselben sind wie die übrigen zu erklären. Borowikow bemerkt ausdrücklich in seinem Beispiele, dass die Neigung zum Zurückfallen erst nach Steigerung des Hirndrucks aufgetreten sei.

Unter 101 Ponstumoren wurde demnach bei 20 cerebellare Ataxie beobachtet. Bei verschiedenen von diesen wurde eine Betheiligung oder Compression des Kleinhirns direct gesehen. Da sämmtliche mit erheblichen Druckerscheinungen einhergingen, war eine Druckwirkung auf das Kleinhirn oder das verlängerte Mark bei allen wahrscheinlich. Bei keinem Falle ist diese Möglichkeit zu verneinen.

Man hat ausserdem zu berücksichtigen, dass bei Erweichungsherden im Pons, die ihre Nachbarschaft in Ruhe lassen, cerebellare Ataxie fehlt. Wenn also diese Ataxie bei Ponstumoren auftritt, kann man sie mit ziemlich grosser Bestimmtheit auf Rechnung nicht der Ponsläsion selbst, sondern des Kleinhirns oder des verlängerten Markes setzen. In unserem Falle kommt nur das letztere in Betracht. Der N. vestibularis, der Deiters'sche Kern, das Corp. restif., woran man dabei denken könnte, wurden allerdings intact befunden.

Herrn Professor Rieger erlaube ich mir meinen aufrichtigen Dank für die liebenswürdige Ueberlassung dieses Falles auszusprechen.

\section{Literatur.}

1) W. Weygandt, Arch. f. Psych. Bd. 28. Heft 2.

2) Spina, Wiener klin. Woch. 1897.

3) Zanke, Neurol. Centralbl. 1897. S. 882.

4) Delbanco, Inaug.-Diss. Berlin 1890.

5) Wiersma, Neurol. Centralbl. 1900. S. 728.

6) Oppenheim, Die Geschwülste des Gehirns. 1897.

7) Bernhardt, Hirngeschwülste. 1881.

8) Nothnagel, Topische Diagnostik der Gehirnkrankheiten 1879.

9) Kolisch, Neurol. Centralbl. 1893. S. 561.

10) Diller, l. id.

11) Hofma'nn, Neurol. Centralbl. 1897. S. 417.

12) Borowikow, Neurol. Centralbl. 1898. S. 226.

13) Handford, ebenda. S. 817.

14) Strözewski, Neurol. Centralbl. 1900. S. 719. 